\title{
CORRESPONDENCIA ENTRE LOS COMPONENTES DEL CURRÍCULO DE ESTUDIOS DE LA ESCUELA DE AGRONOMÍA DE LA FACULTAD DE CIENCIAS AGRÍCOLAS DE LA UNIVERSIDAD NACIONAL "JORGE BASADRE GROHMANN" DE TACNA
}

Nelly Arévalo Solsol ; Everth Castro y Céspedes

\begin{abstract}
$R E S U M E N$
El presente trabajo de investigación se llevó a cabo para determinar si existía correspondencia entre los componentes del curriculo (objetivos generales, perfil profesional, plan de estudios y silabos) de la Escuela Profesional de Agronomía de la Facultad de Ciencias Agrícolas de la Universidad Nacional Jorge Basadre Grohmann de Tacna. También se estudió el nivel de percepción de los estudiantes y docentes que vienen aplicando el mencionado currículo en el año académico 2002.

Las conclusiones a que se han llegado nos permite señalar que existe parcial correspondencia entre los componentes del currículo de la Escuela Profesional de Agronomía.

Los resultados de este trabajo de investigación nos lleva a considerarlos como un anticipo y fuente de información para la auto evaluación que debe hacer la Escuela de Agronomía con fines de acreditación.
\end{abstract}

\section{A B S T R A C T}

This present research work was carried out to determine if there existed correspondence among the components of the curriculum (general objectives, professional background, study plans and syllabus) from the Agronomy Professional School in the faculty of Agriculture Science of the JBG National University of Tacna. The level of perception of the students and lectures who are applying this curriculum in the 2002 academic year was also studied.

The conclusions to which we have carried allow us to point out that there is partial correspondence among the components of the Agronomy Professional School curriculum.

The results of this research work lead us to consider them as a previous stage and resource of information for the self evaluation that the school has to do for acreditation aims.

\section{CAPITULOI}

\section{EL PROBLEMA}

Actualmente la propuesta educativa frente a la realidad pide dotar al futuro Ingeniero Agrónomo de un bagaje sólido en el ámbito cultural, humanístico y ético con conocimientos cientifico - técnico en la producción agrícola y manejo de agro ecosistemas y el desarrollo rural de acuerdo a las exigencias socioeconómicas y culturales de la población, así como crear estrategias y métodos de intervención, cooperación, análisis y reflexión.

Es por esta razón que se debe considerar en la formación del Ingeniero Agrónomo un currículo que sea guía del proceso educativo que tenga sus propositos traducidos en fines, politicas, misiones, visiones, etc. y en donde se formule claramente los objetivos curriculares, el perfil profesional, plan de estudios, los silabos y la evaluación curricular de acuerdo a las caracteristicas antes mencionadas y que debe haber correspondencia entre los diferentes componentes de currículo.

En la Facultad de Ciencias Agricolas, desde e año 1999 se viene aplicando un nuevo currículo, ya que el anterior llamado (curriculo antiguo), está en plena desactivación, con la última promoción que egresa e año 2002. Estando a cuatro años de la aplicación de un nuevo currículo y para alcanzar la "excelencia académica", nos vemos en la necesidad de evalua

(1) MFSc. Mejoramiento Genético de Plantas

(2) MSc. Producción Agricola 
este curriculo para introducirle los reajustes necesarios y asi tomar las mejores decisiones para reestructurarlo si fuera necesario, por lo que nos hemos propuesto con este trabajo de investigación establecer la relación que existe entre el perfil profesional, el plan de estudios y los sillabos de la carrera, por cuanto se piensa que no existe correspondencia entre ellos.

Sobre la base de esta situación se decide investigar: La correspondencia entre los componentes del currículo de estudios de la Escuela Profesional de Agronomía de la Facultad de Ciencias Agrícolas de la Universidad Nacional Jorge Basadre Grohmann de Tacna como son: Los objetivos, el perfil profesional; el plan de estudios y los sílabos.

\subsection{OBJETIVOS DE LA INVESTIGACIÓN:}

\section{Objetivo general:}

Determinar la correspondencia que existe entre los componentes del currículo (objetivos generales, perfil profesional, plan de estudios y sílabos) de la Escuela de Agronomía de la Facultad de Ciencias Agrícolas de la UNJBG - Tacna, así como el nivel de percepción de los estudiantes y docentes que vienen aplicando el mencionado currículo en el año académico 2002.

\section{Objetivos especificos:}

a) Determinar la correspondencia que existe entre los objetivos generales del currículo con el perfil profesional de la Escuela de Agronomía de la FCAG de la UNJBG. - Tacna.

b) Determinar la correspondencia que existe entre el perfil profesional y el plan de estudios de la Escuela de Agronomía de la Facultad de Ciencias Agrícolas de la UNJBG - Tacna.

c) Determinar la correspondencia que existe entre el perfil profesional y los sílabos de la Escuela de Agronomia de la Facultad de Ciencias Agrícolas de la UNJBG - Tacna.

\subsection{HIPÓTESIS DEL TRABAJO:}

Ho: No existe correspondencia entre los componentes del currículo de estudios de la Escuela de Agronomía de la FCAG de la UNJBB - Tacna.

$\mathrm{Ha}$ : Existe correspondencia entre los componentes del currículo de estudios de la Escuela de Agronomía de la FCAG de la UNJBG-Tacna.

\subsection{VARIABLES DE ESTUDIO:}

$$
\begin{aligned}
& \text { Variable : } \text { Perfil profesional } \\
& \text { Indicadores: } \text { - Función de producción } \\
& \text { - Función administrativa }
\end{aligned}
$$

- Función de investigación

- Función docente

Variable : Plan de estudios
Indicadores : - Cursos de formación básica
- Cursos de formación complementaria
- Cursos de formación profesional
- Cursos de especialidad

Variable : Silabo

Indicadores : - Sumilla

- Objetivos

- Contenidos

- Metodología de enseñanza

- Tiempo asignado (horas teoria- horas prácticas)

- Sistema de tvaluación

- Bibliografia

\section{CAPITULO II}

\section{METODOLOGIAA DE LA INVESTIGACIÓN}

\subsection{TIPO DE INVESTIGACIÓN}

\section{Descriptiva y explicativa.}

Es descriptiva porque analiza y describe los diferentes componentes del currículo, al destacar las caracteristicas preponderantes del trabajo educativo y concretamente en el desarrollo curricular de la estructura curricular de la Escuela Profesional de Agronomia de la Facultad de Ciencias Agrícolas de la Universidad Nacional Jorge Basadre Grohmann.

Es explicativa porque trata de identificar las causas o factores que inciden en la adecuada correspondencia que debe existir entre los diferentes componentes que integran el currículo de la Escuela de Agronomia de la Facultad de Ciencias Agricolas de la Universidad Nacional Jorge Basadre Grohmann.

\section{2. ÁMBITO DE ESTUDIO}

La presente invessigación se realizó en la Escuela de Agronomia de la Facultad de Ciencias Agrícolas de la Universidad Nacional Jorge Basadre Grohmann de Tacna, que fue creada en 1979 por Resolución Rectoral 
№ 79-UNJBG. Inicialmente funcionó como Programa Académico de Agronomía (1980) bajo un currículo de sistema flexible-semestral. En 1990 se puso en ejecución un currículo de sistema anual. Desde 1999 se viene aplicando un sistema de currículo semestral rígido con matricula anual. Actualmente la Facultad de Ciencias Agrícolas cuenta con 3 Escuelas Académicas- Profesionales: Agronomía, Economia Agrícola y Medicina Veterinaria y Zootecnia. La Facultad tiene una población de 596 estudiantes.

El docurnento curricular vigente, en su primer capítulo, establece la justificación del currículo de estudios y dentro de ella se diseña las estrategias. En el segundo capítulo trata sobre el perfil profesional y el tercer capítulo se presenta las bases de la estructura curricular y el diseño del plan de estudios.

De acuerdo a las características del presente estudio se tomará como referencia al documento curricular y especificamente perfil profesional, los objetivos curriculares, el plan de estudio y silabos de la Escuela de Agronomía de la Facultad de Ciencias Agrícolas de la UNJBG de Tacna.

\subsection{POBLACIÓN Y MUESTRA}

\subsubsection{Población}

\section{Estuvo constituida por:}

* 21 Profesores de la Escuela Profesional de Agronomia de la UNJBG.

* 178 Estudiantes de la Escuela Profesional de Agronomia de la UNJBG.

* 65 Sillabos de curso correspondientes al plan de estudios de la Escuela de A.gronomía de la UNJBG.

* El perfil profesional de la Escuela Profesional de Agronomía. UNJBG.

* Los objetivos curriculares die la Escuela profesional de Agronomía de la UNJBG.

\subsubsection{Muestra}

La muestra estuvo constituida por 110 alumnos seleccionados al azar estratificado, por 15 profesores, La estructura curricular de la Escuela Profesional de Agronomía de la UNJBG, compuesta por sus objetivos, el perfil profesional, el plan de estudios y 43 sílabos.

\subsection{PROCEDIMIENTO Y RECOLECCIÓN DE INFORMACIÓN}

\subsubsection{Procedimiento}

La recolección de información se realizó utilizando la técnica de la encuesta a estudiantes y docentes para conocer la percepción que tenian sobre el curriculo, componentes y correspondencia.. Asimismo el análisis de los objetivos curriculares y su correspondencia con el perfil profesional y los demás componentes del curriculo fue a nivel personal y de análisis documental, teniendo como apoyo las encuestas aplicadas.

\subsubsection{Técnicas}

Se utilizó la encuesta, aplicada a los estudiantes y docentes, a fin de recoger información sobre la aplicación y desarrollo del currículo y la correspundencia entre sus componentes. Asimismo se utilizó la técnica del análisis documental para establecer la correspondencia entre el perfil profesional y los demás componentes del currículo.

\subsubsection{Instrumentos}

Se titilizó un cuestionario dirigido a los estudiantes y a las docentes para medir el nivel de percepción que tienen acerca del currículo y sus componentes, y la correspondencia que existe entre ellos.

Se usó una ficha de análisis de los silabos (análisis documental) elaborada personalmente, para establecer la correspondencia interna entre los elementos del sillabo así como su correspondencia con el perfil profesional.

\section{Se utilizó una matriz cuadrática:}

- Para evaluar la correspondencia entre las caracteristicas del perfil profesional y las funciones de desempeño del Ingeniero Agrónomo.

- Para evaluar la correspondencia entre las caracteristicas del perfil profesional y el plan de estudios de la Escuela de Agronomia.

- Para evaluar el perfil profesional y los objetivos de los siliabos de los cursos.

Las escalas utilizadas fueron las siguientes:

$1=$ No correspondencia (menor de $25 \%$ )

$3=$ Farcial correspondencia $(26-74 \%)$

5 = Si correspondencia (a partir del 75\%) 


\subsection{PLAN DE ANÁLISIS}

\subsubsection{Formas de análisis:}

Los datos recogidos a través de la aplicación de los cuestionarios dirigidos a los estudiantes, docentes y obtenidos en forma personal, fueron analizados a través de la elaboración de cuadros y gráficas estadisticos que permitieron brindar una explicación cuantitativa y cualitativa de los datos registrados.

La correspondencia entre los componentes del currículo fueron analizados a través de la matriz cuadrática, estableciendo su relación entre sus diferentes elementos (componentes), usando cuadros y gráficos.

\subsubsection{Procedimientos generales que se} utilizaron para someter a prueba las Hipótesis:

Los análisis estadísticos permitieron encontrar la correspondencia existente entre los componentes del currículo, por lo tanto también nos permiten la comprobación de las hipótesis de trabajo.

\subsubsection{Medidas estadisticas que se emplearon:}

El análisis de la información, se hizo en base a la elaboración de cuadros porcentuales y de tendencia central, que permitieron una evaluación clara, objetiva y válida de los datos recogidos.

\section{LOS RESULTADOS}

\section{PLAN DE ESTUDIOS ESAG}

CUADRO N ${ }^{\circ} 01$

ANÁLISIS DEL PLAN DE ESTUDIOS DE LA ESCUELA DE AGRONOMIA DE FCAG - 2002

\begin{tabular}{|l|c|c|}
\hline & & \\
\hline - Asignatura Básica (AB) & 14 & 21,54 \\
\hline - Asignaturas de Formación Profesional (AFP) & 23 & 35,38 \\
\hline - Asignaturas de Formación Especializada (AFE) & 19 & 29,23 \\
\hline - Asignaturas Complementarias (AC) & 09 & 13,85 \\
\hline FOTAtt: Plan de Estudios de la Escuela de Agroñomia 19960,00 \\
\hline
\end{tabular}

GRÁFICA N ${ }^{\circ} 01$

ANÁLISIS DEL PLAN DE ESTUDIOS DE LA ESCUELA DE AGRONOMIA DE FCAG-2002

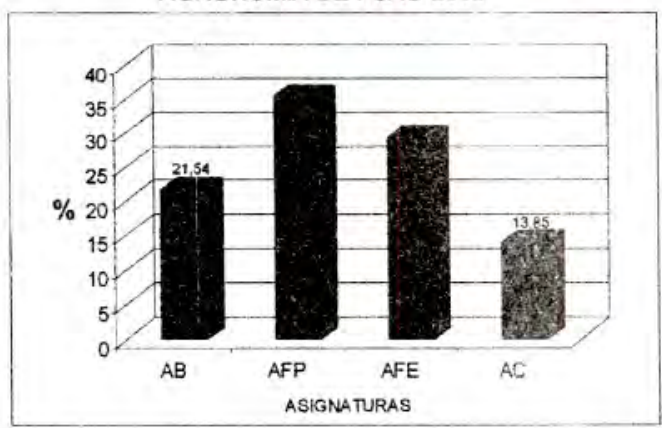

\section{INTERPRETACIÓN}

En el Cuadro $\mathrm{N}^{\circ} 01$ se puede observar el $35,38 \%$ de los cursos que integran el plan de estudios corresponde a la formación profesional del Ingeniero Agrónomo; el 29,23\% corresponde a los cursos de formación especializada; el 21,54\% corresponden a los cursos de formazión básica o humanística; y el $13,85 \%$ representan los cursos de formación complementaria.

Teniendo en cuenta la proporcionalidad porcentual en que se ha distribuido el conjunto de cursos que integran el pian de estudios, podemos afirmar que existe una distribución equitativa y racional donde los pesos asignados a cada uno de los aspectos (formación básica, formación profesional, formación especializada y formación complementaria), es lógico y racional; pero lo que no existe es la correspondencia entre las características o rasgos del perfil profesiona con la naturaieza de cada uno de los cursos del plan de estudios (cuadro $\mathrm{N}^{\circ} 18$ ), asimismo muchos aspectos de estas caracteristicas no han sido tomadas en cuenta, tal como se demuestra, más adelante (cuadro $\mathrm{N}^{\circ} 19$ y gráficas $\mathrm{N}^{\circ} 19$ y 20 ), donde se hace un análisis más minucioso de la correspondencia entre el perfil profesional y los objetivos por cada uno de los sílabos de los cursos de la Carrera Profesional de Agronomía. Da la impresión que en la elaboración del plan de estudios, y su distribución a lo largo de los cinco años de estudios que dura la Carrera de Formación del Ingeniero Agrónomo, no se tomó en cuenta lo que establecia el perfil profesional de dicha carrera. Ya qus de las once caracteristicas que conforma el perfil profesional, solamente dos rasgos han sido tomados en cuenta ya que la mayorla de objetivos apurita hacia ellos; y existen rasgos que no han sido cor siderados como la formación de valores, el desarroilo autónomo del futuro profesional, la formación critica y creativa.

\subsection{ANÁLISIS: EVALUACIÓN DE LOS SÍLABOS}

CUADRO N ${ }^{\circ} 02$

CONTENIDOS PRINCIPALES EN LA SUMILLA -2002

\begin{tabular}{|l|c|c|}
\hline Se aprecia & & \\
\hline En parte & 34 & 79,07 \\
\hline No se aprecia & 4 & 9,30 \\
\hline TOTAL & 5 & 11,63 \\
\hline
\end{tabular}

Fuente: Silabos Esculuela de Agronomla 2002 


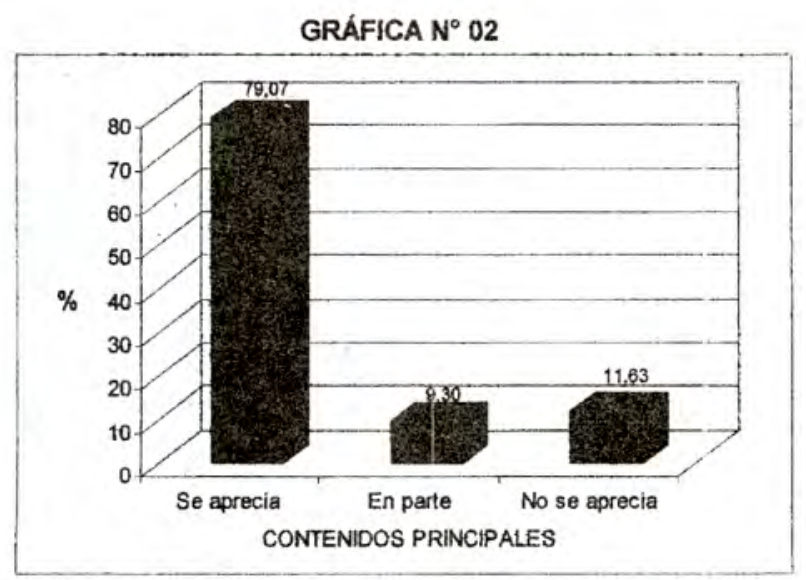

Fuente: Cuadro $\mathrm{N}^{\circ} \mathrm{O2}$

\section{INTERPRETACIÓN}

En el Cuadro $\mathrm{N}^{\circ} 07$, en cuanto a los contenidos principales del curso, al analizar los sillabos encontramos que en el $79,07 \%$, sí se aprecian en forma clara, precisa y concisa dichos contenidos; no así en el $9,30 \%$ que se observa en parte; y el $11,63 \%$ donde no se observa con claridad dichos contenidos

Al evaluar la existencia de la claridad en relación a los objetivos (conducta, contenido, patrón de rendimiento) encontramos las siguientes observaciones:

\section{CUADRO $\mathrm{N}^{\circ} 03$}

CLARIDAD DE LOS OBJETIVOS DE LOS SILABOS DE LOS CURSOS -2002

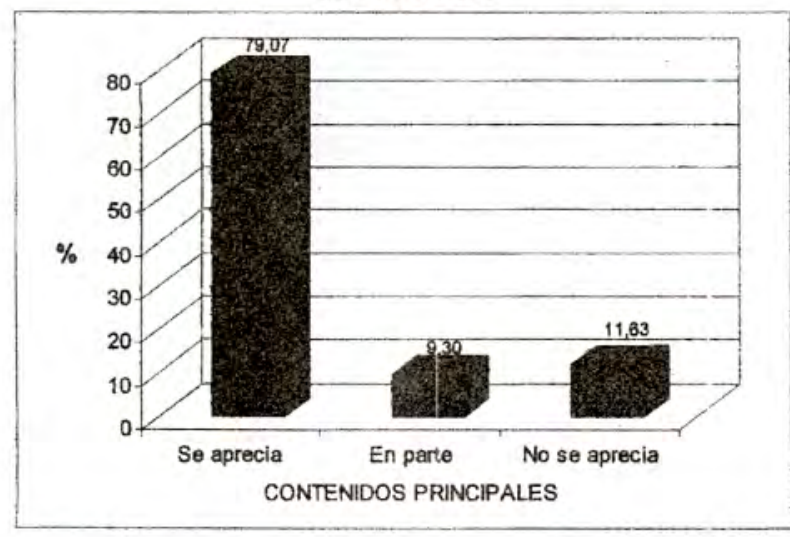

Fuente: Cuadro $\mathrm{N}^{\circ} 03$

\section{INTERPRETACIÓN}

En el Cuadro $\mathrm{N}^{\circ} \mathrm{O3}$, en cuanto a los contenidos principales del curso, al analizar los sillabos encontramos que en el $79,07 \%$, si se aprecian en forma clara, precisa y concisa dichos contenidos; no asi en el $9,30 \%$ que se observa en parte; y el $11,63 \%$ donde no se observa con claridad dichos contenidos
Al evaluar la existencia de la claridad en relación a los objetivos (conducta, contenido, patrón de rendimiento) encontramos las siguientes observaciones:

\section{CUADRO N 04}

CLARIDADDE LOS OBJETIVOS DELOS SILLABOS DELOS CURSOS -2002

\begin{tabular}{|l|c|c|}
\hline Sí & & \\
\hline En parte & 23 & 53,49 \\
\hline No & 18 & 41,86 \\
\hline TOTAL & 2 & 4,65 \\
\hline
\end{tabular}

Fuente: Silabos Escuela de Agronomía 2002

GRÁFICA N 04

CLARIDAD DE LOS OBJETIVOS DE LOS SÍLABOSS DE LC ${ }^{\circ}$ CURSOS -2002

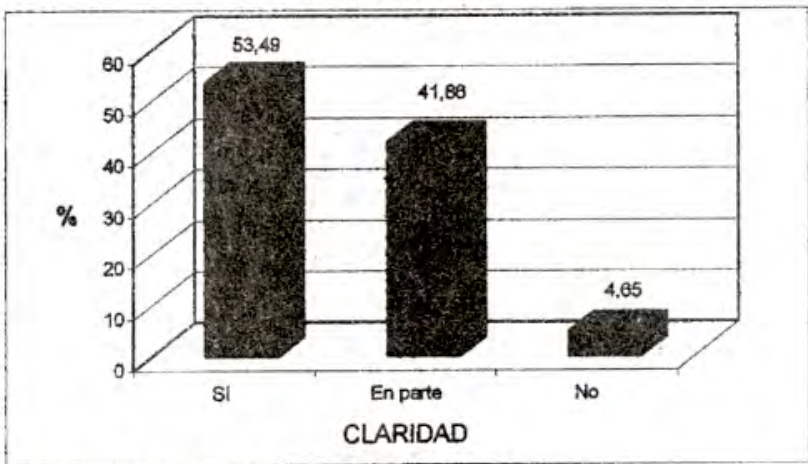

Fuente: Cuadro $\mathfrak{N}^{\circ} 04$

\section{INTERPRETACIÓN}

En el Cuadro $\mathrm{N}^{\circ} 04$ se observa que el $53,49 \%$ de ios sílabos tienen objetivos claros; el 41,86 se aprecia una claridad relativa; y el 4,65 no tiene claridad en la redacción de los objetivos. Este aspecto es importante dentro de la estructuración de los sílabos, porque, si el docente no tiene claro los objetivos que persigue el curso, este es desarrollado a la deriva en forma mecánica y repetitiva, sin ningún horizonte y menos pensando en tener una relación con las características del perfil profesional.

Planteamiento de la metodologia teniendo en cuenta el principio de diversidad de la metodologia:

\section{CUADRO N ${ }^{\circ} 05$}

METODOLOGIA DIVERSIFICADA-2002

\begin{tabular}{|l|c|c|}
\hline Si & 33 & 76.74 \\
\hline En parte & 7 & 16.28 \\
\hline No & 3 & 6.98 \\
\hline TOTAL & 43 & 100,00 \\
\hline
\end{tabular}


GRÁFICA N ${ }^{\circ} 05$

METODOLOGIA DIVERSIFICADA-2002

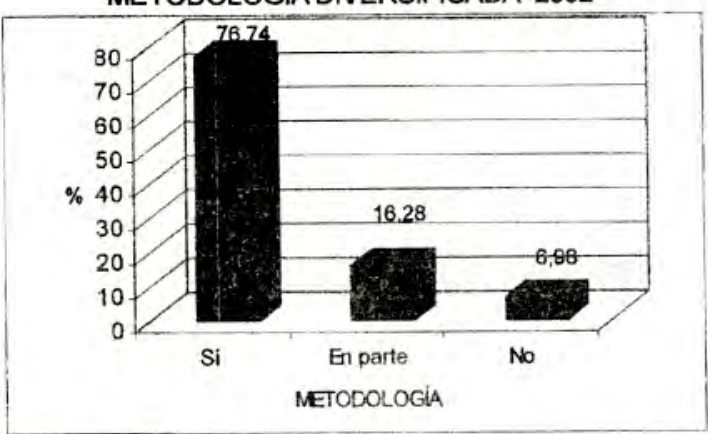

Fuente: Cuadro $\mathrm{N}^{\circ} 05$

\section{INTERPRETACIÓN}

En el Cuadro $N^{\circ} 11$, al evaluar este aspecto (planteamiento de la metodología) se observa que el $76,74 \%$ de los docentes programan una metodología diversificada, esto dando cumplimiento a las normas establecidas por la Facultad, sin embargo hay que resaltar que el $16,28 \%$ solamente tiene en cuenta en parte este principio; y el $6,98 \%$ ni siquiera lo tiene en cuenta.

Actualización de la bibliografía de los silabos:

\section{CUADRO Nº6}

ACTUALIZACIÓN BIBLIOGRÁFICA DE LOS SILABOS -2002

\begin{tabular}{|l|c|c|}
\hline & & \\
\hline Si & 4 & 9,30 \\
\hline En parte & 25 & 58,14 \\
\hline No & 14 & 32,56 \\
\hline TOTAL & $\mathbf{4 3}$ & $\mathbf{1 0 0 , 0 0}$ \\
\hline
\end{tabular}

Fuente: Silabos Escuela de Agronomia 2002

GRÁFICA N ${ }^{\circ} 06$

ACTUALIZACIÓN BIBLIOGRÁFICA DE LOS SILABOS -2002

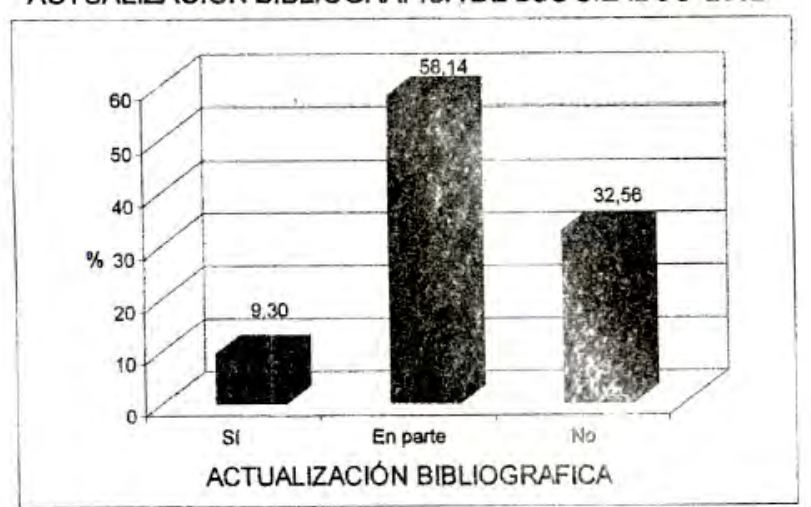

Fuente: Cuadro $\mathrm{N}^{\circ} 13$

\section{INTERPRETACIÓN}

En el Cuadro $\mathrm{N}^{\circ} 13$, se observa que el $58,14 \%$ de los sillabos presenta una bibliografía relativamente actualizada, es decir con una antigüedad aceptable (periodo de 10-15 años de antigüedad); el 32,56\% presenta una bibliografía desactualizada, es decir mayor de 15 años de antigüedad; y solamente el 9,30\% está actualizada, estos resultados son muy preocupantes ya que teniendo en cuenta el avance de la ciencia y la tecnología y la rapidez de estos cambios, está bibliografía debería permanentemente actualizarse.

Correspondencia interna entre los elementos del sílabo (sumilla, objetivos, contenidos, metodologia, evaluación, bibliografia):

\section{CUADRO $N^{\circ} 07$}

CORRESPONDENCIA INTERNA ENTRE LOS ELEMENTOSDEL SILABO -2002

\begin{tabular}{|l|c|c|}
\hline Si & & 24 \\
\hline En parte & 19 & 55.81 \\
\hline No & 0 & 44.19 \\
\hline TOTAL & 43 & 0,00 \\
\hline
\end{tabular}

Fuente: Silabos Escuela de Agronomia 2002

\section{GRÁFICA N ${ }^{\circ} 07$}

CORRESPONDENCIA INTERNAENTRELOSELEMENTOSDEL SILABO -2002

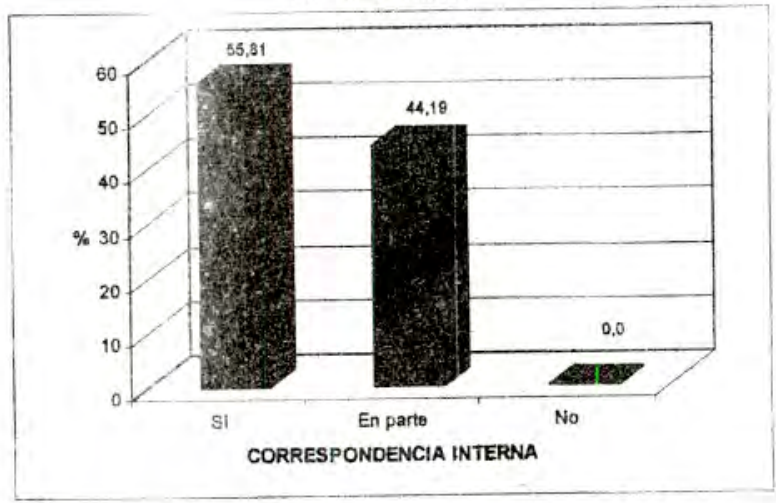

Fuente: Cuadro $N^{\circ} 07$

\section{INTERPRETACIÓN}

En el Cuadro $\mathrm{N}^{\circ} 15$, al evaluar la correspondencia interna entre los elementos del sílabos se observa, que solo el $44,19 \%$ tiene una correspondencia en parte, y el $55,81 \%$ tiene correspondencia.

Resultados de la Evaluación Global a los sílabos de la Escuela de Agronomia de la Facultad de Ciencias Agricolas. 
CUADRO N 08

EVALUACIÓN GLOBAL DE LOS SIILABOS DE LAESCUELADE AGRONOMIADE LAFACULTAD DE CIENCIAS AGRICOLAS 2002

\begin{tabular}{|l|c|c|}
\hline & & \\
\hline & & \\
\hline Si & 15 & 34,88 \\
\hline Parcial & 26 & 60,47 \\
\hline No & 2 & 4,65 \\
\hline TOTAL & 43 & 100,00 \\
\hline
\end{tabular}

Fuente: Silabos Escuela de Agronomía 2002

\section{GRÁFICA N ${ }^{\circ} 08$}

EVALUACION GLOBAL DE LOS SÍLABOS DE LAESCUELADE AGRONOMIA DE LAFACULTAD DE CIENCIAS AGRICOLAS 2002

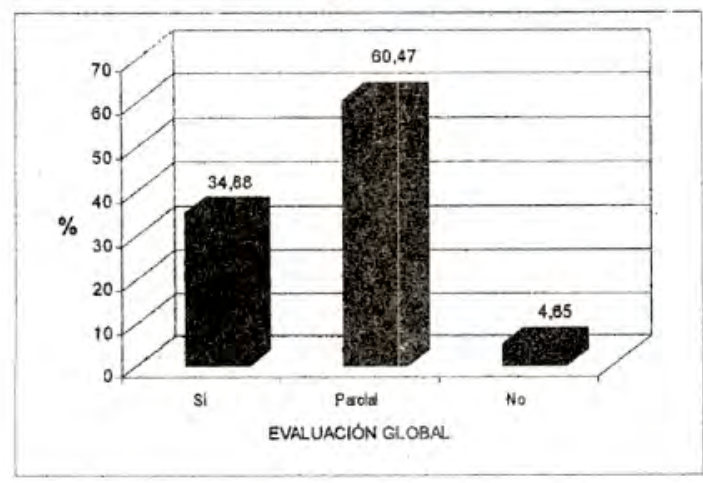

Fuente: Cuadro $N^{\circ} 08$

\section{INTERPRETACIÓN}

En el Cuadro $N^{\circ} 08$, al evaluar los sílabos para detectar el grado de correspondencia con el perfil profesional, los resultados indican que el $60,47 \%$ tiene parcial correspondencia, el $34,88 \%$ tiene correspondencia y el $4,65 \%$ no tiene ninguna correspondencia. Estos resultados confirman nuestra hipótesis planteada en que no existe correspondencia entre el perfil profesional y los sílabos de la Escuela de Agronomia de la FCAG de la UNJBG; la cual representa el $65,12 \%$.

\subsection{CORRESPONDENCIA ENTRE EL PERFIL PROFESIONAL DE LA ESCUELA DE AGRONOMÍA Y LAS FUNCIONES DEL DESEMPEÑO DEL INGENIERO AGRÓNOMO}

\section{INTERPRETACIÓN}

Al realizar la correspondencia entre el perfil profesional y el desempeño del Ingeniero Agrónomo, se puede apreciar que existe una marcada correspondencia directa, observándose que esta correspondencia es equitativa y lógica, dando prioridad a las funciones de producción e investigación del futuro Ingeniero Agrónomo, sin descuidar las otras funciones de administración y docente; por lo que se ve, que el perfil profesional de la Carrera de Agronomia opunta una formación integral del futuro Ingeniero Agrónomo: es decir que el perfil profesional es bueno; sin embargo cuando analizamos la correspondencia del perfil profesional con el plan de estudios no se aprecia esta correspondencia (Cuadro $\mathrm{N}^{\circ} 18$ ); asímismo, podemos ver que, al analizar los silabos existe una correspondencia parcial (Cuadro $\mathrm{N}^{\circ} 16$ ); el silabo del curso es elaborado por el profesor en forma individual y mal, sin tener presente el perfil profesional y es el sílabo el que repercute en el rendimiento académico tal como se aprecia en el (cuadro 24 y gráficas $\mathrm{N}^{\circ} 25$ y 26 ).

\section{CUADRO N 09}

MATRIZDE CORRESPONDENCIAENTREEL PERFIL PROFESIONAL YLAS FUNCIONES DEL DESEMPEÑO DEL INGENIEROAGRONOMO

\begin{tabular}{|c|c|c|c|c|c|c|c|c|c|c|c|c|}
\hline \multirow{3}{*}{ Perfil profesional } & \multicolumn{12}{|c|}{ FUNCIONES } \\
\hline & \multirow{2}{*}{ F } & \multicolumn{3}{|c|}{ rmaduccios } & \multicolumn{3}{|c|}{ Nvistancicen } & \multicolumn{2}{|c|}{ Dockstr } & \multicolumn{3}{|c|}{ apresstrucios } \\
\hline & & & & 1. & & $.1 \mathrm{~T}$. & 51 & & $7 \times 1$, & & & \\
\hline Un profesional integral y competitivo en las condiciones socio económica y culturales del pais. & 4 & 1 & 1 & & \begin{tabular}{l|l}
1 & 1 \\
\end{tabular} & & 1 & 11 & & 1 & 1 & \\
\hline $\begin{array}{l}\text { 2. Conciente y motivado para el autoestudio y la capacitación permanente de acuerdo a la realidad y al } \\
\text { avance cientifico tecnológico. }\end{array}$ & 4 & 1 & 1 & & 11 & 1 & 1 & 11 & & 1 & 1 & \\
\hline 3. Critico y con capacidad de reorientar las estrategias del agro peruano. & 1 & & & & & & & & & 1 & 1 & \\
\hline $\begin{array}{l}\text { 4. Practicar y promover una agricultura sostenible privilegiando las tecnologias tendientes a la preservación, } \\
\text { conservación, y utilización racional de los recursos naturales. }\end{array}$ & 2 & 1 & 1 & & & & & & & 1 & 1 & \\
\hline $\begin{array}{l}\text { Creativo con una visión amplia y que actúe con eficacia en la explotación agropecuaria en el incremento } \\
\text { de la producción y la productividad. }\end{array}$ & 2 & 1 & 1 & & 1 & 1 & & & & & & \\
\hline Práctica de los valores éticos y morales. & 4 & 1 & 1 & & 1 & 1 & & 11 & & 1 & 1 & \\
\hline Capaz de transmitir tecnologías aplicando estrategias adecuadas para su ejecución gradual. & 2 & 1 & 1 & & 1 & 1 & & & & & & \\
\hline $\begin{array}{l}\text { 8. Profesional lider con mentalidad exupresarial, tendiente a lograr una producción agroindustrial y de } \\
\text { exportacion. }\end{array}$ & 2 & 1 & 1 & & & & & & & 1 & 1 & \\
\hline Capaz de aplicar la informática al desarrollo de la agricultura. & 2 & & & & 1 & 1 & & 1 & 1 & & & \\
\hline Que sean capaces de integrarse en grupos de trabajo (multisectorial). & 4 & 1 & 1 & & 1 & 1 & & 11 & & 1 & 1 & \\
\hline Profesional capaz de planificar, organizar, dirigir, desarrollar y evaluar la investigación cientifica. & 2 & & & & 1 & 1 & & 1 & 1 & & & \\
\hline 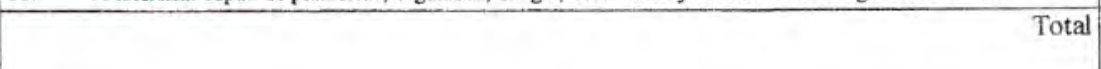 & 29 & & 8 & & & 62 & & 64 & 2 & 7 & 7 & \\
\hline$\%$ & 100 & & $\frac{1}{27,5}$ & & & 27,59 & & & 0,69 & & 24 , & 13 \\
\hline
\end{tabular}




\subsection{CORRESPONDENCIA ENTRE EL PERFIL PROFESIONAL Y EL PLAN DE ESTUDIOS DE LA ESCUELA DE AGRONOMÍA DE LAFACULTAD DE CIENCIAS AGRICOLAS}

\section{CUADRO $N^{\circ} 18$}

MATRIZ DE CORRESPONDENCIAENTRE EL.PERFIL PROFESIONAL Y EL PLAN DE ESTUDIOS DELAESCUELADEAGRONOMIADE LA FACULTAD DE CIENCIASAGRICOLAS

\begin{tabular}{|c|c|c|c|c|c|c|c|}
\hline \multirow[b]{2}{*}{ Características del Perfil profesional } & \multicolumn{7}{|c|}{ ASIGNATURAS } \\
\hline & $\mathrm{F}$ & Sí corresp. & $\%$ & $\begin{array}{l}\text { Parcial } \\
\text { corresp. }\end{array}$ & $\%$ & No corresp. & $\%$ \\
\hline $\begin{array}{l}\text { 1. Un profesional integral y competitivo en las condiciones socio } \\
\text { económica y culturales del pais. }\end{array}$ & 12 & 7 & 7,70 & 5 & 5,49 & 0 & 0 \\
\hline $\begin{array}{l}\text { 2. Conciente y motivado para el autoestudio y la capacitación } \\
\text { permanente de acuerdo a la realidad y al avance cientifico } \\
\text { tecnológico. }\end{array}$ & 1 & 0 & 0 & 1 & 1,10 & 0 & $\theta$ \\
\hline $\begin{array}{l}\text { 3. Critico y con capacidad de reorientar las estrategias del agro } \\
\text { peruano. }\end{array}$ & 1 & 0 & 0 & 1 & 1,10 & 0 & 0 \\
\hline $\begin{array}{l}\text { 4. Practicar y promover una agricultura sostenible privilegiando las } \\
\text { tecnologias tendientes a la preservación, conservación, y } \\
\text { utilización racional de los recursos naturales. }\end{array}$ & 11 & 8 & 8,80 & 3 & 3,30 & 0 & 0 \\
\hline $\begin{array}{l}\text { 5. Creativo con una visión amplia y que actúe con eficacia en la } \\
\text { explotación agropecuaria en el incremento de la producción y la } \\
\text { productividad. }\end{array}$ & 39 & 34 & 37,36 & 5 & 3,48 & 0 & 0 \\
\hline 6. Práctica de los valores éticos y morales. & 0 & 0 & 0 & 0 & 0 & 0 & 0 \\
\hline \begin{tabular}{|l|l|} 
Capaz de transmitir tecnologias aplicando estrategias adecuadas \\
para su ejecución gradual.
\end{tabular} & 6 & 0 & 0 & 6 & 6,58 & 0 & 0 \\
\hline $\begin{array}{l}\text { 8. Profesional líder con mentalidad empresarial, tenciente a lograr } \\
\text { una producción agroindustrial y de exportación. }\end{array}$ & 11 & 4 & 4,40 & 7 & 7,69 & 0 & 0 \\
\hline 9. Capaz de aplicar la informatica al desarrollo de ia agricultura. & 1 & 0 & 0 & $i$ & 1,10 & 0 & 0 \\
\hline $\begin{array}{l}\text { 10. Que sean capaces de integrarse en grupos de trabajo } \\
\text { (multisectorial). }\end{array}$ & 3 & 0 & 0 & 3 & 3,30 & 0 & 0 \\
\hline $\begin{array}{l}\text { 11. Profesional capaz de planificar, organizar, dirigir, desarrollar y } \\
\text { evaluar la investigación cientifica. }\end{array}$ & 6 & 4 & 4,40 & 2 & 2,20 & 0 & 0 \\
\hline TOTAL & 91 & 57 & & 34 & & 0.0 & \\
\hline$\%$ & 100 & & 62,64 & & 37,36 & & 0,0 \\
\hline
\end{tabular}

Fuente: Plan de estudios de la Escuela de Agronomía de la FCAG.

\section{INTERPRETACIÓN}

El perfil profesional de la Carrera de Agronomia de Facultad de Ciencias Agrícolas está constituida por 11 caracteristicas, y el plan de estudios está integrada por 65 cursos.

Al efectuar la correspondencia entre el perfil profesional y el plan de estudios (Cuadro $N^{\circ} 18$ ) se puede observar que la mayoria de asignaturas están dirigidas a la característica 5 "creativo con una visión amplia y que actué con eficacia en la explotación agropecuaria en el incremento de la procucción y la productividad", con una correspondencia directa del $37,36 \%$ y parcialmente con $5,48 \%$, le sigue ia característica 4 "Practicar y promover una agricultura sostenible privilegiando las tecnologías tendientes a la preservación, conservación, y utilización racional de los recursos naturales", con una correspondencia directa de $8,80 \%$ y parcialmente $30,30 \%$; así mismo la caracteristica 1 "Un profesional integral y competitivo en las condiciones socio económica y culturales del país", con una correspondencia directa del $7,70 \%$ y de $5,49 \%$ de correspondencia parcial; asímismo, se pueden mencionar a la característica 8 "Profesional lider con mentalidad empresarial, tendiente a lograr una producción agroindustrial y de exportación".

También podemos visualizar, que en el plan de estudios la característica 6 "Práctica de los valores éticos y morales", no tiene un curso que justifique está característica.

Como se puede apreciar el plan de estudios de la Escuela de Agronomía tiene una correspondencia parcial la cual está dirigida a cuatro características $(5,4,1$ y 8$)$; por lo que se puede decir, que las demás características sólo han sido consideradas débilmenie. Si comparamos estos resultados con las del cuadro № 19 y gráfica N$^{\circ} 20$ se puede decir que hay una significativa relación con respecto a la caracteristica 5. 


\subsection{CORRESPONDENCIA ENTRE EL PERFIL PROFESIONAL DE LA ESCUELA DE AGRONOMIA YLOS OBJETIVOS DE LOS SÍLABOS DE LOS CURSOS - AÑO 2002}

CUADRO N 10

CORRESPONDENCIA ENTRE EL PERFIL PROFESIONAL DE LA ESCUELA DE AGRONOMIA YLOS OBJETIVOS DE LOS SILABOS DE LOS CURSOS - ANNO 2002

\begin{tabular}{|c|c|c|c|c|c|c|c|}
\hline \multirow{2}{*}{ Caracteristicas del Perfil profesional } & \multicolumn{7}{|c|}{ ORIETIVOS DE LOS SILABOS DE CURSOS } \\
\hline & $\mathrm{F}$ & Si corresp. & $\%$ & $\begin{array}{l}\text { Parcial } \\
\text { carresp. }\end{array}$ & $\%$ & No & $\%$ \\
\hline $\begin{array}{l}\text { 1. Un profesional integral y competitivo on las condiciones socio } \\
\text { económica y culturales del pais. }\end{array}$ & 10 & 0 & 0 & 6 & 2,92 & 4 & 1,95 \\
\hline $\begin{array}{l}\text { 2. Conciente y motivado para el autoestudio y la capacitación } \\
\text { permanante de acuerdo a la realiclad y al avance científico } \\
\text { tecnológico. }\end{array}$ & 8 & 1 & 0,48 & 2 & 0,97 & 5 & 2,50 \\
\hline $\begin{array}{l}\text { 3. Crítico y con capacidad de recrientar las estratogias del agro } \\
\text { peruana }\end{array}$ & 17 & 2 & 0,97 & 13 & 6,34 & 2 & 0,97 \\
\hline $\begin{array}{l}\text { 4. Practicar y promover una agricultura scotenible privilegiando las } \\
\text { tecnologias tendientes a la presarvación, conservación, y } \\
\text { utilización racional de los recursos naturales. }\end{array}$ & 14 & I & 0,48 & 13 & 6,34 & 0 & 0 \\
\hline $\begin{array}{l}\text { 5. Creativo con una visión amplia y que actíe con eficacia en la } \\
\text { explotación agropecuaria en el incremento de la producción y la } \\
\text { productividad. }\end{array}$ & 100 & 6 & 2,92 & 93 & 45,37 & 1 & 0,49 \\
\hline 5. Práctica de los valores éticos y mornles. & 26 & 4 & $1, \overline{95}$ & 21 & 10,24 & 1 & 0,49 \\
\hline $\begin{array}{l}\text { 7. Capaz de transmitir tocnologias aplicando estratogias adecuadas } \\
\text { para su ejecución gradual. }\end{array}$ & 11 & 0 & 0 & 11 & 5,37 & 0 & 0 \\
\hline $\begin{array}{l}\text { 8. Profesional lider con mentalidad empresarial, tendiente a lograr } \\
\text { una producción agroindustrial y de exportación. }\end{array}$ & 3 & 1 & 0,18 & 2 & 0,97 & 0 & 0 \\
\hline 9. Capaz de aplicar la informática al desarrollo de la agricultura. & 0 & 0 & 0 & 0 & 0 & 0 & 0 \\
\hline $\begin{array}{l}\text { 10. Que sean capaces de integrarse an grupos de trabajo } \\
\text { (multisoctorial). }\end{array}$ & 3 & 0 & 0 & 3 & 1,46 & 0 & 0 \\
\hline $\begin{array}{l}\text { 11. Profesional capaz de planificar, crganizar, dirigir, desarrollar y } \\
\text { evaluar la investigación cientifica. }\end{array}$ & 13 & 4 & 1,95 & 9 & 4,36 & 0 & 0 \\
\hline TOTAL & 205 & 19 & & 173 & & 13 & \\
\hline$\%$ & 100 & & 9,23 & & 84,37 & & 6,40 \\
\hline
\end{tabular}

Fuente: Silabos de cursos - 2002 y perfil profesional de la Escuela de Agronomia

\section{INTERPRETACIÓN}

Para encontrar la correspondencia entre las características del perfil profesional, con los objetivos de los sílabos pertenecientes a cada uno de los cursos de la Escuela Profesional de Agronomía hacemos el siguiente análisis (Cuadro $\mathrm{N}^{\circ} 10$ ).

El total de sílabos de los cursos analizados suman (43) que viene a ser el número de cursos perteneciente al currículo de la Carrera de Agronomia (currículo vigente).

Se han analizado 205 objetivos entre generales y específicos, pertenecientes a la totalidad de asignaturas señaladas anteriormente que vendrian a constituir el $100 \%$ de objetivos analizados, de este total se ha encontrado que un $9,27 \%$, guarda correspondencia con algunas de las características del perfil profesional, pero un tanto significativo lo tenemos en la correspondencia parcial que existe entre los objetivos de los sílabos de los cursos con las características del perfil profesional ya que representa un $84,39 \%$ del total de objetivos analizados. Como se puede ver el nivel de correspondencia que existe entre las características del perfil profesional con los objetivos de los silabos tiene una parcial correspondencia. Hay que hacer notar que producto del análisis de los objetivos de los sílabos de los cursos analizados existe un $6,34 \%$ de objetivos que no guardan ninguna correspondencia con alguna caracteristica del perfil profesional, hecho que nos muestra de que es un dato muy significativo dentro del presente análisis.

Es necesario resaltar en el presente análisis, que de la tutalidad de objetivos 126 objetivos $(61,46 \%)$ se concentra en la característica 5, del perfil profesional, características que está dirigida a "tener una visión amplia y que actúe con eficacia en la explotación agropecuaria en el incremento de la producción y la productividad", olvidándose que el perfil profesional de su carrera comprende también otras características de formación, tales como una visión amplia sobre la situación económica actual del país, motivado para una autoformación; crítico y creativo de su propia realidad, el desarrollo de capacidades para la investigación, etc. Es decir, existe una correspondencia parcial entre las características del perfil profesional con los objetivos de cursos y éstos se encuentran en una característica, solamente, descuidando el resto de caracteristicas de la formación del futuro profesional de agronomía.

\section{CONCLUSIONES}

\section{Primera Conclusión:}

Existe parcial comespondencia entre los componentes del curículo de la Escuela Profesional de Agronomía de la Facultad de Ciencias Agrícolas de la Universidad Nacional Jorge Basadre Grohmann de Tacna. 


\section{Segunda Conclusión:}

Al no visualizarse los objetivos generales del curriculo en los documentos curriculares de la Carrera de Agronomía, por no estar determinados en forma clara y precisa, sino estar presentados a través de las estrategias establecidas para la formación del Ingeniero Agrónomo, no se ha podido establecer la correspondencia con el perfil profesional; motivando que exista una serie de incongruencias entre los diferentes componentes del currículo, teniendo en cuenta que la determinación de los objetivos generales del currículo es el aspecto fundamental para definir toda la planeación educativa y curricular.

\section{Tercera Conclusión:}

Existe parcial correspondencia entre el perfil profesional y el plan de estudios de la carrera de agronomia, tal como se ha demostrado a través de los análisis realizados a lo largo del presente trabajo de investigación.

\section{Cuarta Conclusión:}

Existe parcial correspodencia entre el perfil profesional y los objetivos del silabo, esto se aprecia tanto en el planeamiento de los objetivos curriculares como las estrategias sugeridas y la bibliografia consignada.

\section{REFERENCIABIBLIOGRÁFICA}

1. Arista, M. G. (1976). Currículum I. EDU-SMP. Lima-Perú. 2. Arnaz, A. J. (1996): La Planeación Curricular. 2da Edición. Editorial Trillas. México.

3. Asamblea Nacional de Rectores (Comisión Nacional de Rectores para la Acreditación. (2003). Propuesta de Estándares Mínimos para Autoevaluación con fines de Acreditación de Carreras de Ingeniería. Impreso por la Oficina de Acreditación e Investigación de la ANR. Lima-Perú.

4. Bloon, B. (1992): Taxonomía de objetivos de la Educación. Editorial Ateneo. México.

5. Capella, J.R. (1991): Una década de la Educación Peruana 1980 - 1990. Reflexiones y propuestas. Ed. Cultura y Desarrollo. 1ra. Ed. Lima-Perú.

6. Chiroque, S. (2001): Pedagogía y Didáctica Universitaria. Seminario Taller UNJBG - Tacna-Perú.

7. Diaz Barriga, A. F. y otros (1993): Metodología de Diseño curricular para Educación Superior. Editorial Trillas, México. 8. Díaz Barriga, Á. (1992): Ensayos sobre la Problemática Curricular. $4^{\mathrm{a}}$. Edición, Editorial Trillas Limusa, México.

9. Gago, A. (1977): Modelos de Sistematixación del Proceso Enseñanza-Aprendizaje. Edit. Trillas. México. 10. Glazman, E. E lbarrola de M. (1978). Diseños de Planes de Estudio. CISE-UNAM. México.

11. Garrido, F. Rodríguez, G. (1979). Diseños de Planes de Estudio. Plan de Trabajo. Tesis de Licenciatura. Facultad de Psicología. UNAM.México.

12. Hernández Sampieri, R. y otros (1997). Metodología de la Investigación. Mc Graw Hill. Bogotá-Colombia.
13. Instituto Nacional de Investigación Educativa (INIDE). (1980). Curriculum 1. Fundamentos de Planificación Curricular (Antología). Segunda Ed. Lima-Perú.

14. Ley General de Educación $N^{\circ} 28044$. (2003). Diario Oficial "El Peruano". Lima. Perú.

15. Leyton Soto, M. y Tyler, R. W. (1972). Planeamiento Educacional (Princípios Básícos del Currículum y del Aprendizaje. Un Modelo Pedagógico) Segunda Edición. Edit. Universitaria. Santiago de Chite.

16. Marrou, A. (1997): Elaboración de un Programa Analítico, Curso de Post grado en Pedagogia Universitaria (Separata). UNJBG Tacna.

17. Mestas, M. (1997): Teoría del Currículo. Universidad Nacional de San Agustin de Arequipa. Facultad de Educación. Arequipa Perú.

18. Ministerio de Educación del Perú (Comisión Técnica de Currículum).(1976). Estudios Básicos sobre el Currículo en el Sistema Educativo Peruano. Impreso. INIDE. Lima-Perú.

19. Pacheco V. A. y colaboradoras (1988). Didáctica Universitaria. 1ra Edición. Universidad de Lima, Facultad de Ciencias Humanas. Lima-Perú

20. Pontificia Universidad Católica del Perú (1987) Lineamientos Básicos para el Trabajo Curricular Documento de Trabajo del Centro de investigaciones y Servicios Educativos (CISE-PUC). Lima-Perú.

21. Posner, J. G. (1998). Análisis del Currículo. 2da Edición. Edit. Mc Graw Hill. Bogotá-Colombia.

22. Rivera, A. (1988): Currículo y Aprendizaje. Editorial San Marcos, Lima Perú.

23. Rodriguez, R. M. (1994). Estructura Curricular Aplicable a la Maestria en Educación. Revista Educación. Año II. N². Lima-Perú.

24. Rodríguez, V. E. (2003). Currículo. Modulo I. Curso de Post Grado. VIAD-OASA. Universidad Nacional Jorge Basadre Grohmann. Separatas.

25. Rossi, Q. E. (1989). Teoría y Técnica Curricular. Edit. Universitaria. San martín de Porres. Lima-Perú

26. Taba, H. (1982): Elaboración del currículo. Editorial Troquela Argentina.

27. Ticona, L. (1996): Evaluación Curricular: Un Punto de Visita de Docentes y Estudiantes. Tesis Escuela de Post Grado, Universidad Privada de Tacna.-Perú,

28. Tyler, R.W. (1979). Principios Básicos del Currículum. Troquel. Buenos Aires. Argentina.

29. Universidad Nacional Jorge Basadre Grohmann de Tacna (1999). Currículo de Estudios de la Escuela Académico Profesional de Agronomia. Impreso por la INJBG. Tacna-Perú 30. Valente, R.C. (1997): Propuesta y Análisis del Currículo de la Facultad de Obstetricla de la UNJBG de Tacna. Tesis Escuela de Post Grado. Universidad Privada de Tacna. Perú 